\title{
A NOTE ON SETS OF CONSTANT WIDTH
}

\section{Z. A. MELZAK}

1. Let $E_{n}$ be the $n$-dimensional Euclidean space and let $K$ be a bounded closed convex set in $E_{n}$. For any direction $u$ there are exactly two supporting hyperplanes of $K$, orthogonal to $u$, and the distance $D_{u}(K)$ between them is called the width of $K$ in the direction $u$. By a hyperplane we always mean one of dimension $n-1$.

$K$ is called a set of constant width 1 , or briefly of width 1 , if $D_{u}(K)=1$ for all $u$. A sphere $S_{r}(x)$ denotes the closed solid sphere in $E_{n}$ of radius $r$ about the center $x . S_{r}(x)$ is the circum-sphere (the in-sphere) of $K$ if $K \subset S_{r}(x)\left(S_{r}(x) \subset K\right)$ and the radius $r$ is smallest (largest) possible. We shall prove the following

THeORem 1. Let $K \subset E_{n}$ be a set of width 1. Then $K$ has a unique circum-sphere $S_{R}(p)$, a unique in-sphere $S_{r}(q)$ and

(1) $1-[n / 2(n+1)]^{1 / 2} \leqq r, R \leqq[n / 2(n+1)]^{1 / 2}$,

(2) $r+R=1$,

(3) $p=q$.

Further, in both estimates of (1) we have either the strict inequality together or equality together, and the latter occurs if and only if $K$ contains a unit regular n-simplex.

In the proof free use will be made of the standard properties of convex sets in general and sets of constant width in particular. For these see the standard reference [1].

2. The existence of a unique circum-sphere $S_{R}(p)$ follows at once from the observation that the intersection of two spheres of equal radii and distinct centers is a subset of a sphere of smaller radius.

The existence of a unique $r$ such that $S_{r}(q)$ is the in-sphere of $K$ for some $q$ or $q$ 's follows from a simple compactness argument and we have

$$
r=\max _{x \in K} \min _{y \in \mathbb{B}(K)}|x y|
$$

where $B(K)$ is the boundary of $K$. Suppose now that there are two in-spheres $S_{r}\left(q_{1}\right)$ and $S_{r}\left(q_{2}\right), q_{1} \neq q_{2}$. It follows then that $S_{r}\left(q_{3}\right)$ is also an in-sphere for any $q_{3}$ on the open segment $\left(q_{1} q_{2}\right)$; therefore the set $V=B(K) \cap S_{r}\left(q_{3}\right)$ is not empty, let $t \in V$. Let also $H$ be the hyperplane through $t$ orthogonal to $t q_{3}$. Then $H$ is a supporting hyperplane both of $S_{r}\left(q_{3}\right)$ and $K$. Since $H$ cannot intersect the interiors of $S_{r}\left(q_{1}\right)$

Received by the editors July 20, 1959. 
and $S_{r}\left(q_{2}\right)$ it follows that $H$ supports both these spheres. If we let $x_{i}=H \cap S_{r}\left(q_{i}\right)$ then the straight segment $x_{1} x_{2}$ lies in $B(K)$. However, the boundary of a set of constant width cannot contain straight segments, or in other words, every point of it is extremal. This leads to a contradiction and so $q_{1}=q_{2}=q$ say. We have proved the existence and uniqueness of both spheres.

It is a consequence of the definition of constant width that if $H_{1}$ and $\mathrm{H}_{2}$ are two distinct parallel supporting hyperplanes of $K$ and $x_{1}=H_{1} \cap ß(K), x_{2}=H_{2} \cap ß(K)$, then $\left|x_{1} x_{2}\right|=1$ and $x_{1} x_{2}$ is orthogonal to $H_{1}$ and $H_{2}$. Let $x, y, z$ be three collinear points such that $y \in K$ $-B(K)$ is fixed and $x, z \in B(K)$ are variable. It follows from the previous observation that if $|x y|$ is minimum then $|y z|$ is maximum and conversely, while in either case $|x z|=1$. As a consequence we have

$$
S_{1-R}(p) \subset K \subset S_{1-r}(q)
$$

which implies that $1-R \leqq r$ and $R \leqq 1-r$, proving (2).

By the theorem of Jung $[2 ; 3]$ we have

$$
R \leqq[n / 2(n+1)]^{1 / 2} .
$$

This together with (2) implies (1). (3) is a simple corollary since by (4) and (2) $S_{r}(p) \subset K$ and so by the uniqueness of the in-sphere $p=q$. This concludes the proof of the first part of our theorem.

3. We show next that if $K$ contains a unit regular $n$-simplex $T$ and has the circum-sphere $S_{R}(p)$, then $R=[n / 2(n+1)]^{1 / 2}$ and so by (2) $r=1-[n / 2(n+1)]^{1 / 2}$. Further, $p$ is the center of $T$.

Let $T$ have vertices $v_{1}, \cdots, v_{n+1}$ and center $o$. We denote by $x y$ the vector from $x$ to $y$ and by $|x y|=(x y \cdot x y)^{1 / 2}$ its length. We have

$$
\sum_{1}^{n+1} o v_{j}=0
$$

and

$$
o v_{i} \cdot o v_{j}=c, \quad i \neq j, \quad\left|o v_{i}\right|=k .
$$

Squaring (5) and making use of (6) we get

$$
k^{2} \sum_{1}^{n+1} 1+2 c \sum_{i<j} 1=0
$$

or

$$
k^{2}+n c=0 .
$$


Since $\left|v_{i} v_{j}\right|=1$ for $i \neq j$, we also have

$$
\left(o v_{i}-o v_{j}\right) \cdot\left(o v_{i}-o v_{j}\right)=1
$$

and, multiplying this out,

$$
2 k^{2}-2 c=1
$$

Now by (7) and (8)

$$
c=o v_{i} \cdot o v_{j}=-1 / 2(n+1), \quad k=[n / 2(n+1)]^{1 / 2} .
$$

Suppose now that $p \neq o$, then it is easy to show that for some $v_{i}$

$$
\left|p v_{i}\right|>\left|o v_{i}\right|=k=[n / 2(n+1)]^{1 / 2} .
$$

However, this contradicts Jung's theorem. Therefore $p=o$ and $R=k$ $=[n / 2(n+1)]^{1 / 2}$.

4. It remains to be shown that if a set $K$ of width 1 has the circumsphere $S_{R}(o)$ and $R=[n / 2(n+1)]^{1 / 2}$ then $K$ contains a unit regular $n$-simplex. Since $K$ is convex it suffices to show that $K$ contains the $n+1$ vertices of such simplex. We need first a few lemmas. Let $X(K)=ß(K) \cap \otimes\left[S_{R}(o)\right]$; it is easy to show that $X(K)$ contains at least two distinct points.

Lemma 1. If $S_{R}(o)$ is the circum-sphere of $K$ and $R=[n / 2(n+1)]^{1 / 2}$ then for $x_{1}, x_{2} \in X(K), x_{1} \neq x_{2}$, we have

$$
o x_{1} \cdot o x_{2} \geqq-1 / 2(n+1) \text {. }
$$

By the definition of $X(K)$ we have

$$
o x_{1} \cdot o x_{1}=o x_{2} \cdot o x_{2}=R^{2}=n / 2(n+1)
$$

and

$$
\left|x_{1} x_{2}\right|^{2}=\left(o x_{1}-o x_{2}\right) \cdot\left(o x_{1}-o x_{2}\right) \leqq 1 .
$$

Hence by multiplying out

$$
2 R^{2}-2 o x_{1} \cdot o x_{2} \leqq 1
$$

which gives at once (10).

Lemma 2. Under the assumptions of Lemma 1 the set $X(K)$ contains at least $n+1$ points.

Let $X(K)=\left\{x_{1}, \cdots, x_{j}\right\}$. It is easily shown that $o$ lies in the closed convex hull $\mathrm{e}(X)$ of $X=X(K)$. Therefore there exist numbers $\alpha_{1}, \cdots, \alpha_{j}$ such that 


$$
0 \leqq \alpha_{i}, \quad \sum_{1}^{j} \alpha_{i}=1, \quad \sum_{1}^{j} \alpha_{i} 0 x_{i}=0 .
$$

Squaring the last equation and observing that $o x_{i} \cdot o x_{i}=R^{2}$ we have

$$
R^{2} \sum_{1}^{j} \alpha_{i}^{2}+2 \sum_{i<k} \alpha_{i} \alpha_{k} o x_{i} \cdot o x_{k}=0,
$$

and by Lemma 1 and the hypothesis that $R^{2}=n / 2(n+1)$, we get

$$
\sum_{1}^{j} \alpha_{i}^{2} \leqq(2 / n) \sum_{i<k} \alpha_{i} \alpha_{k}
$$

Since $\sum_{1}^{j} \alpha_{i}=1$ by squaring we get

$$
\sum_{1}^{j} \alpha_{i}^{2}=1-2 \sum_{i<k} \alpha_{i} \alpha_{k}
$$

By comparing (11) and (12) we obtain

$$
n / 2(n+1) \leqq \sum_{i<k} \alpha_{i} \alpha_{k}
$$

and this together with (12) yields

$$
\sum_{1}^{j} \alpha_{i}^{2} \leqq 1 /(n+1)
$$

However, given $j$ nonnegative numbers $\alpha_{1}, \cdots, \alpha_{j}$ whose sum is 1 , the sum of their squares attains its minimum $1 / j$ for $\alpha_{1}=\cdots=\alpha_{j}$ $=1 / j$. Hence by (13) $1 / j \leqq 1 /(n+1)$ and so $j \geqq n+1$, which proves the lemma.

Since $o \in \mathfrak{C}(X)$ and by Lemma $2 X=X(K)$ contains at least $n+1$ points, there exists a subset $U$ of $X$ such that $U=\left\{x_{1}, \cdots, x_{n+1}\right\}$ and $o \in \mathfrak{C}(U)$.

Lemma 3. The points $x_{1}, \cdots, x_{n+1}$ are vertices of a unit regular $n$ simplex inscribed into $S_{R}(o)$.

Repeating the procedure of Lemma 2 we have

$$
\sum_{1}^{n+1} \alpha_{i} o x_{i}=0
$$

where $0 \leqq \alpha_{i}$ and $\sum_{1}^{n+1} \alpha_{i}=1$. Moreover, reasoning as before, we get

and therefore

$$
\sum_{1}^{n+1} \alpha_{i}^{2}=1 /(n+1)
$$




$$
\alpha_{1}=\alpha_{2}=\cdots=1 /(n+1)
$$

so that by (14)

$$
\sum_{1}^{n+1} o x_{i}=0 .
$$

Squaring this and recalling that $o x_{i} \cdot o x_{i}=R^{2}=n / 2(n+1)$, we have

$$
R^{2} \sum_{1}^{n+1} 1+2 \sum_{i<k} o x_{i} \cdot o x_{k}=0
$$

or

$$
\sum_{i<k} o x_{i} \cdot o x_{k}=-n / 4 \text {. }
$$

Now, there are $n(n+1) / 2$ numbers $o x_{i} \cdot o x_{k}, i<k$. By the above their sum is $-n / 4$ and so their arithmetic mean is $-1 / 2(n+1)$. Hence by (10) we get finally

$$
o x_{i} \cdot o x_{k}=-1 / 2(n+1), \quad{ }_{k}
$$

Now the lemma follows at once from (15) and (16).

An examination of the proofs of Lemmas 1-3 shows that no use has been made of the property of constant width. In fact, these lemmas together with Jung's theorem can be stated as an independent proposition which is actually an extension of Jung's result:

THEOREM 2. A set of diameter 1 has a circum-sphere of radius $R \leqq[n / 2(n+1)]^{1 / 2}$ and the equality holds if and only if $K$ contains the vertices of a unit regular $n$-simplex.

This concludes the proof of Theorem 1.

5. The author wishes to thank the referee and Professor B. A. Rattray of McGill University for their illuminating criticism and suggestions.

Added in proof. The author has recently found out that parts of Theorem 1 have been proved by H. G. Eggleston in his Cambridge University Tract on Convexity.

\section{REFERENCES}

1. T. Bonnesen and W. Fenchel, Konvexe Körper, reprinted by Chelsea, 1950.

2. H. W. E. Jung, Über die kleinste Kugel die eine räumliche Figur einschliesst, J. Reine Angew. Math. vol. 123 (1901) pp. 241-257.

3. D. Gale, On inscribing n-dimensional sets in a regular $n$-simplex, Proc. Amer. Math. Soc. vol. 4 (1953) pp. 222-225. 